\title{
On the almost sure central limit theorem for self-normalized products of partial sums of $\phi$-mixing random variables
}

Kyo-Shin Hwang ${ }^{*}$

\section{"Correspondence:}

hwang0412@naver.com

Department of Mathematics,

Zhejiang University, Yuquan

Campus, Hangzhou, 310027,

P.R. China

RINS, College of Natural Science, Gyeongsang National University, Jinju, 660-701, Korea

\begin{abstract}
Let $\left\{X_{n}, n \geq 1\right\}$ be a sequence of strictly stationary $\phi$-mixing positive random variables which are in the domain of attraction of the normal law with $E X_{1}=\mu>0$, possibly infinite variance and mixing coefficient rates $\phi(n)$ satisfying $\sum_{n \geq 1} \phi^{1 / 2}\left(2^{n}\right)<\infty$. Under suitable conditions, we here give an almost sure central limit theorem for self-normalized products of partial sums, i.e.,

$$
\lim _{n \rightarrow \infty} \frac{1}{D_{n}} \sum_{m=1}^{n} d_{m} l\left(\left(\prod_{k=1}^{m} \frac{S_{k}}{k \mu}\right)^{\mu /\left(\beta V_{m}\right)} \leq x\right)=F(x) \quad \text { a.s. for any } x \in R,
$$
\end{abstract}

where $F$ is the distribution function of the random variable $e^{\sqrt{2} \mathcal{N}}$ and $\mathcal{N}$ is a standard normal random variable.

MSC: $60 \mathrm{~F} 15$

Keywords: almost sure central limit theorem; $\phi$-mixing; domain of attraction of the normal law; self-normalized product of partial sums; strictly stationary

\section{Introduction and main results}

The almost sure central limit theorem (ASCLT) was first introduced independently by Brosamler [1] and Schatte [2]. Since then, many interesting results have been discovered in this field. The classical ASCLT states that when $E X=0, \operatorname{Var}(X)=\sigma^{2}$,

$$
\lim _{n \rightarrow \infty} \frac{1}{\log n} \sum_{k=1}^{n} \frac{1}{k} I\left\{\frac{S_{k}}{\sqrt{k} \sigma} \leq x\right\}=\Phi(x) \quad \text { a.s. for any } x \in R
$$

Here and in the sequel, $I\{\cdot\}$ denotes an indicator function and $\Phi(\cdot)$ is the distribution function of the standard normal random variable. It is known (see Berkes [3]) that the class of sequences satisfying the ASCLT is larger than the class of sequences satisfying the central limit theorem. In recent years, the ASCLT for products of partial sums has received more and more attention. We refer to Gonchigdanzan and Rempala [4] on the ASCLT for the products of partial sums, Gonchigdanzan [5] on the ASCLT for the products of partial sums with stable distribution. Li and Wang [6] and Zhang et al. [7] showed ASCLT for products of sums and products of sums of partial sums under association. Huang and Pang [8], Zhang and Yang [9] obtained the ASCLT results of self-normalized versions. 
Zhang and Yang [9] proved the following ASCLT for self-normalized products of sums of i.i.d. random variables.

Theorem A Let $\left\{X, X_{n}, n \geq 1\right\}$ be a sequence of i.i.d. positive random variables with $\mu=$ $E X>0$, and assume that $X$ is in the domain of attraction of the normal law. Then

$$
\lim _{n \rightarrow \infty} \frac{1}{\log n} \sum_{k=1}^{n} \frac{1}{k} I\left(\left(\prod_{j=1}^{k} \frac{S_{j}}{j \mu}\right)^{\mu / V_{k}} \leq x\right)=F(x) \quad \text { a.s. for any } x \in R,
$$

where $F(\cdot)$ is the distribution function of the random variable $e^{\sqrt{2} \mathcal{N}}$ and $\mathcal{N}$ is a standard normal random variable.

A wide literature concerning the ASCLT of self-normalized versions of independent random variables is now available, while the ASCLT for self-normalized versions of weakly dependent random variables is worth studying. Recalling that $\left\{X_{n}, n \geq 1\right\}$ is a sequence of random variables and $\mathcal{F}_{a}^{b}$ denotes the $\sigma$-field generated by the random variables $X_{a}, X_{a+1}, \ldots, X_{b}$. The sequence $\left\{X_{n}, n \geq 1\right\}$ is called $\phi$-mixing if

$$
\phi(n)=\sup _{k \geq 1} \sup _{A \in \mathcal{F}_{1}^{k}, B \in \mathcal{F}_{k+n}^{\infty}}|P(B \mid A)-P(B)| \rightarrow 0 \quad \text { as } n \rightarrow \infty .
$$

The sequence $\left\{X_{n}, n \geq 1\right\}$ is called $\rho$-mixing if

$$
\rho(n)=\sup _{k \geq 1} \sup _{\xi \in L_{2}\left(\mathcal{F}_{1}^{k}\right), \eta \in L_{2}\left(\mathcal{F}_{k+n}^{\infty}\right)} \frac{|\operatorname{Cov}(\xi, \eta)|}{\left(E \xi^{2}\right)^{1 / 2}\left(E \eta^{2}\right)^{1 / 2}} \rightarrow 0 \quad \text { as } n \rightarrow \infty,
$$

where $L_{2}\left(\mathcal{F}_{a}^{b}\right)$ is a set of all $\mathcal{F}_{a}^{b}$-measurable random variables with second moments. It is well known that $\rho(n) \leq 2 \phi^{1 / 2}(n)$, and hence a $\phi$-mixing sequence is $\rho$-mixing.

Theorem B (Balan and Kulik [10,11]) Let $\left\{X_{n}, n \geq 1\right\}$ be a strictly stationary $\phi$-mixing sequence of nondegenerate random variables such that $E X_{1}=0$ and $X_{1}$ belongs to the domain of attraction of the normal law. Let $S_{n}=\sum_{i=1}^{n} X_{i}$ and $\bar{V}^{2}=\sum_{i=1}^{n} X_{i}^{2}$. Suppose that $\phi(1)<1$ and the mixing coefficients $\phi(n)$ satisfy $\sum_{n \geq 1} \phi^{1 / 2}\left(2^{n}\right)<\infty$, then

$$
\text { (i) } \quad \frac{S_{n}}{\bar{A}_{n}} \stackrel{d}{\rightarrow} \mathcal{N}(0,1) \quad \text { and } \quad \frac{\bar{V}_{n}}{\bar{B}_{n}} \stackrel{p}{\rightarrow} 1
$$

where

$$
\bar{A}_{n}^{2}=\operatorname{Var}\left(\sum_{i=1}^{n} X_{i} I\left\{\left|X_{i}\right| \leq \tau_{i}\right\}\right), \quad \bar{B}_{n}^{2}=\sum_{i=1}^{n} \operatorname{Var}\left(X_{i} I\left\{\left|X_{i}\right| \leq \tau_{i}\right\}\right),
$$

and $\tau_{i}=\inf \left\{s: s \geq 1, \frac{L(s)}{s^{2}} \leq \frac{1}{i}\right\}$ for $i=1,2, \ldots$

In this paper we study the almost sure central limit theorem, containing the general weight sequences, for weakly dependent random variables. Let $\left\{X_{n}, n \geq 1\right\}$ be a sequence of strictly stationary $\phi$-mixing positive random variables which are in the domain of attraction of the normal law with $E X_{1}=\mu>0$, possibly infinite variance and mixing coefficients 
$\phi(n)$ satisfying $\sum_{n \geq 1} \phi^{1 / 2}\left(2^{n}\right)<\infty$. We here give an almost sure central limit theorem for self-normalized products of partial sums under a fairly general condition.

Throughout this paper, the following notations are frequently used. For any two positive sequences, $a_{n} \ll b_{n}$ means that for a certain numerical constant $C$ not depending on $n$, we have $a_{n} \leq C b_{n}$ for all $n$, and $a_{n} \sim b_{n}$ means $a_{n} / b_{n} \rightarrow 1$ as $n \rightarrow \infty$. $[x]$ denotes the largest integer smaller or equal to $x$, and $C$ denotes a generic positive constant, whose value can differ in different places.

We let $l(x)=E\left(X_{1}-\mu\right)^{2} I\left\{\left|X_{1}-\mu\right| \leq x\right\}, b=\inf \{x \geq 1: l(x)>0\}$ and

$$
\eta_{n}=\inf \left\{s: s \geq b+1, \frac{l(s)}{s^{2}} \leq \frac{1}{n}\right\}, \quad n=1,2, \ldots
$$

then it is easy to see that $n l\left(\eta_{n}\right) \sim \eta_{n}^{2}$ and $\eta_{n} \leq \eta_{n+1}$ (cf. de la Pena et al. [12]). We denote

$$
A_{n}^{2}=\operatorname{Var}\left(\sum_{j=1}^{n}\left(X_{j}-\mu\right) I\left\{\left|X_{j}-\mu\right| \leq \eta_{n}\right\}\right), \quad B_{n}^{2}=\sum_{j=1}^{n} \operatorname{Var}\left(\left(X_{j}-\mu\right) I\left\{\left|X_{j}-\mu\right| \leq \eta_{n}\right\}\right) .
$$

Our main theorem is as follows.

Theorem 1.1 Let $\left\{X_{n}, n \geq 1\right\}$ be a sequence of strictly stationary $\phi$-mixing positive random variables with $E X_{1}=\mu>0$, possibly infinite variance. Assume that $X_{1}$ belongs to the domain of attraction of the normal law, and the mixing coefficients $\phi(n)$ satisfy $\sum_{n \geq 1} \phi^{1 / 2}\left(2^{n}\right)<\infty$. Denote $S_{n}=\sum_{i=1}^{n} X_{i}$ and $V_{n}^{2}=\sum_{i=1}^{n}\left(X_{i}-\mu\right)^{2}$. If, moreover,

$$
A_{n}^{2} \sim \beta^{2} B_{n}^{2} \quad \text { for some } \beta \in(0, \infty)
$$

then we have

$$
\lim _{n \rightarrow \infty} \frac{1}{D_{n}} \sum_{k=1}^{n} d_{k} I\left(\left(\prod_{j=1}^{k} \frac{S_{j}}{j \mu}\right)^{\mu /\left(\beta V_{k}\right)} \leq x\right)=F(x) \quad \text { a.s. for any } x \in R,
$$

where $F(\cdot)$ is the distribution function of the random variable $e^{\sqrt{2} \mathcal{N}}, \mathcal{N}$ is a standard normal random variable and

$$
d_{k}=k^{-1} \exp \left(\ln ^{\alpha} k\right), \quad 0 \leq \alpha<1 / 2, \quad D_{n}=\sum_{k=1}^{n} d_{k} .
$$

Remark 1.1 If we assume that

$$
\lim _{n \rightarrow \infty} \frac{1}{l\left(\eta_{n}\right)} \sum_{j=2}^{n} \operatorname{Cov}\left(X_{1} I\left\{\left|X_{1}\right| \leq \eta_{n}\right\}, X_{j} I\left\{\left|X_{j}\right| \leq \eta_{n}\right\}\right)=\alpha>-1 / 2,
$$

then $A_{n}^{2} \sim \beta^{2} B_{n}^{2}$ with $\beta^{2}=1+2 \alpha$.

We have the following corollaries.

Corollary 1.1 Let $\left\{X_{n}, n \geq 1\right\}$ be a strictly stationary $\phi$-mixing sequence of positive random variables such that $E X_{1}=\mu>0, \operatorname{Var}\left(X_{1}\right)=\sigma^{2}<\infty$ and $\sum_{j \geq 2}\left|E X_{1} X_{j}\right|<\infty$, then (1.4) holds. 
Corollary 1.2 Let $\left\{X_{n}, n \geq 1\right\}$ be a strictly stationary $\phi$-mixing sequence of positive random variables such that $E X_{1}=\mu>0, \operatorname{Var}\left(X_{1}\right)=\sigma^{2}<\infty$ and $\sum_{n \geq 1} \phi^{1 / 2}\left(2^{n}\right)<\infty$. Set $S_{n}=\sum_{i=1}^{n} X_{i}$ and $\sigma_{n}^{2}=\operatorname{Var}\left(S_{n}^{2}\right)$, then (1.4) holds.

Remark 1.2 Let $d_{k}=1 / k$ and $\beta=1$. If $\left\{X_{n}, n \geq 1\right\}$ is a sequence of i.i.d. positive random variables such that $E X_{1}=\mu>0$ and $X_{1}$ belongs to the domain of attraction of the normal law, then Theorem 1.1 is just Theorem A.

Remark 1.3 By the terminology of summation procedures (see [13, p.35]), Theorem 1.1 remains valid if we replace the weight sequence $\left\{d_{k}\right\}_{k \geq 1}$ by any $\left\{d_{k}^{*}\right\}_{k \geq 1}$ such that $0 \leq d_{k}^{*} \leq$ $d_{k}$ and $\sum_{k \geq 1} d_{k}^{*}=\infty$.

\section{Lemmas}

In this section, we introduce some lemmas which are used to prove our theorem.

Lemma 2.1 (Csörgő et al. [14]) Let $X$ be a random variable, and denote $l(y)=E(X-$ $\mu)^{2} I\{|X-\mu| \leq y\}$. The following statements are equivalent:

(a) $X$ is in the domain of attraction of the normal law,

(b) $y^{2} P\{|X-\mu|>y\}=o(l(y))$,

(c) $y E|X-\mu| I\{|X-\mu|>y\}=o(l(y))$,

(d) $E|X-\mu|^{\alpha} I\{|X-\mu| \leq y\}=o\left(y^{\alpha-2} l(y)\right)$ for $\alpha>2$.

For all positive integers $1 \leq i \leq k<\infty$, we denote

$$
\begin{aligned}
& \widetilde{X}_{i k}=\left(X_{i}-\mu\right) I\left\{\left|X_{i}-\mu\right| \leq \eta_{k}\right\}, \quad \widehat{X}_{i k}=\left(X_{i}-\mu\right) I\left\{\left|X_{i}-\mu\right|>\eta_{k}\right\}, \\
& \widetilde{X}_{i k}^{*}=\widetilde{X}_{i k}-E \widetilde{X}_{i k}, \quad \widehat{X}_{i k}^{*}=\widehat{X}_{i k}-E \widehat{X}_{i k}, \quad b_{i, k}=\sum_{l=i}^{k} \frac{1}{l}, \\
& \widetilde{Y}_{k}=\sum_{i=1}^{k} b_{i, k} \widetilde{X}_{i k}^{*}, \quad \widehat{Y}_{k}=\sum_{i=1}^{k} b_{i, k} \widehat{X}_{i k}^{*}, \quad \widetilde{V}_{k}^{2}=\sum_{i=1}^{k} \widetilde{X}_{i k}^{2} .
\end{aligned}
$$

Lemma 2.2 Let $f$ be a nonnegative, bounded Lipschitz function such that

$$
f(x) \leq C \text { and }|f(x)-f(y)| \leq C|x-y| \quad \text { for every } x, y \in R .
$$

If the assumptions of Theorem 1.1 hold and there exists a positive constant $\epsilon$ such that

$$
\operatorname{Var}\left(\sum_{k=1}^{n} d_{k} f\left(\frac{\tilde{Y}_{k}}{\beta \sqrt{2 k l\left(\eta_{k}\right)}}\right)\right) \ll D_{n}^{2}\left(\ln D_{n}\right)^{-1-\epsilon}
$$

then we have

$$
\lim _{n \rightarrow \infty} \frac{1}{D_{n}} \sum_{k=1}^{n} d_{k} f\left(\frac{\tilde{Y}_{k}}{\beta \sqrt{2 k l\left(\eta_{k}\right)}}\right)=E f(\mathcal{N}(0,1)) \quad \text { a.s. }
$$

Proof From the formula (2.5) in Liu and Lin [15], we have

$$
\frac{\widetilde{Y}_{k}}{\beta \sqrt{2 k l\left(\eta_{k}\right)}} \stackrel{d}{\rightarrow} \mathcal{N}(0,1)
$$


as $k \rightarrow \infty$ under the hypotheses of Theorem 1.1. Then

$$
E f\left(\frac{\widetilde{Y}_{k}}{\beta \sqrt{2 k l\left(\eta_{k}\right)}}\right) \rightarrow E f(\mathcal{N}(0,1))
$$

as $k \rightarrow \infty$, which implies from Toeplitz's lemma that

$$
\frac{1}{D_{n}} \sum_{k=1}^{n} d_{k} E f\left(\frac{\widetilde{Y}_{k}}{\beta \sqrt{2 k l\left(\eta_{k}\right)}}\right) \rightarrow E f(\mathcal{N}(0,1))
$$

as $n \rightarrow \infty$. To prove (2.3), we only need to show that

$$
\lim _{n \rightarrow \infty} \frac{1}{D_{n}} \sum_{k=1}^{n} d_{k}\left[f\left(\frac{\tilde{Y}_{k}}{\beta \sqrt{2 k l\left(\eta_{k}\right)}}\right)-E f\left(\frac{\tilde{Y}_{k}}{\beta \sqrt{2 k l\left(\eta_{k}\right)}}\right)\right]=0 \quad \text { a.s. }
$$

Let

$$
v_{n}=\frac{1}{D_{n}} \sum_{k=1}^{n} d_{k}\left(f\left(\frac{\widetilde{Y}_{k}}{\beta \sqrt{2 k l\left(\eta_{k}\right)}}\right)-E f\left(\frac{\widetilde{Y}_{k}}{\beta \sqrt{2 k l\left(\eta_{k}\right)}}\right)\right) \quad \text { for } n \geq 1
$$

By (2.2), we have

$$
E v_{n}^{2}=\frac{1}{D_{n}^{2}} \operatorname{Var}\left(\sum_{k=1}^{n} d_{k} f\left(\frac{\widetilde{Y}_{k}}{\beta \sqrt{2 k l\left(\eta_{k}\right)}}\right)\right) \ll\left(\ln D_{n}\right)^{-1-\epsilon}
$$

Note that for $\alpha=0$, we get $d_{k}=e / k, D_{n} \sim e \ln n$. For $\alpha>0$, we get

$$
\begin{aligned}
D_{n} & \sim \int_{0}^{\ln n} \exp \left(t^{\alpha}\right) d t \\
& \sim \int_{0}^{\ln n}\left(\exp \left(t^{\alpha}\right)+\frac{1-\alpha}{\alpha} t^{-\alpha} \exp \left(t^{\alpha}\right)\right) d t \\
& =\frac{1}{\alpha}(\ln n)^{1-\alpha} \exp \left(\ln ^{\alpha} n\right),
\end{aligned}
$$

and using Karamata's theorem (see Seneta [16]),

$$
\exp \left(\ln ^{\alpha} x\right)=\exp \left(\int_{1}^{x} \alpha(\ln u)^{\alpha-1} / u d u\right), \quad \alpha<1,
$$

is a slowly varying function at $\infty$. Hence $D_{n+1} \sim D_{n}$. Let $\gamma$ be such that $0<\gamma<\epsilon /(1+\epsilon)$, and $n_{k}=\inf \left\{n: D_{n} \geq \exp \left(k^{1-\gamma}\right)\right\}$, then

$$
D_{n_{k}} \geq \exp \left(k^{1-\gamma}\right)>D_{n_{k}-1}
$$

and thus

$$
1 \leq \frac{D_{n_{k}}}{\exp \left(k^{1-\gamma}\right)} \sim \frac{D_{n_{k}-1}}{\exp \left(k^{1-\gamma}\right)}<1
$$


which means that $D_{n_{k}} \sim \exp \left(k^{1-\gamma}\right)$. Since $(1-\gamma)(1+\epsilon)>1$, we have

$$
\sum_{k=1}^{\infty} E v_{n_{k}}^{2} \leq C \sum_{k=1}^{\infty} \frac{1}{k^{(1-\gamma)(1+\epsilon)}}<\infty
$$

which implies $v_{n_{k}} \rightarrow 0$ a.s. For any given $n$, there exists $k$ such that $n_{k} \leq n<n_{k+1}$. It is easy to see that by the boundedness of $f$,

$$
\left|v_{n}\right| \leq\left|v_{n_{k}}\right|+\frac{1}{D_{n_{k}}} \sum_{i=n_{k}}^{n_{k+1}} d_{i} \leq\left|v_{n_{k}}\right|+C\left(\frac{D_{n_{k+1}}}{D_{n_{k}}}-1\right) \rightarrow 0 \quad \text { a.s., }
$$

which yields (2.5). Hence (2.3) holds true.

Lemma 2.3 Assumef is a nonnegative, bounded Lipschitz function such that $f(x) \leq C$ and $|f(x)-f(y)| \leq C|x-y|$ for every $x, y \in R$. If there exists a positive constant $\epsilon$ such that

$$
\begin{aligned}
& \operatorname{Var}\left(\sum_{k=1}^{n} d_{k} f\left(\frac{\widehat{Y}_{k}}{\beta \sqrt{2 k l\left(\eta_{k}\right)}}\right)\right) \ll D_{n}^{2}\left(\ln D_{n}\right)^{-1-\epsilon}, \\
& \operatorname{Var}\left(\sum_{k=1}^{n} d_{k} f\left(\frac{\widetilde{V}_{k}^{2}}{k l\left(\eta_{k}\right)}\right)\right) \ll D_{n}^{2}\left(\ln D_{n}\right)^{-1-\epsilon}, \\
& \operatorname{Var}\left(\sum_{k=1}^{n} d_{k} I\left\{\bigcup_{i=1}^{k}\left\{\left|X_{i}-\mu\right|>\eta_{k}\right\}\right\}\right) \ll D_{n}^{2}\left(\ln D_{n}\right)^{-1-\epsilon} .
\end{aligned}
$$

Then, under the assumptions of Theorem 1.1, we have

$$
\begin{aligned}
& \lim _{n \rightarrow \infty} \frac{1}{D_{n}} \sum_{k=1}^{n} d_{k} f\left(\frac{\widehat{Y}_{k}}{\beta \sqrt{2 k l\left(\eta_{k}\right)}}\right)=\lim _{k \rightarrow \infty} E f\left(\frac{\widehat{Y}_{k}}{\beta \sqrt{2 k l\left(\eta_{k}\right)}}\right) \text { a.s., } \\
& \lim _{n \rightarrow \infty} \frac{1}{D_{n}} \sum_{k=1}^{n} d_{k} f\left(\frac{\widetilde{V}_{k}^{2}}{k l\left(\eta_{k}\right)}\right)=\lim _{k \rightarrow \infty} E f\left(\frac{\widetilde{V}_{k}^{2}}{k l\left(\eta_{k}\right)}\right) \text { a.s., } \\
& \lim _{n \rightarrow \infty} \frac{1}{D_{n}} \sum_{k=1}^{n} d_{k} I\left\{\bigcup_{i=1}^{k}\left\{\left|X_{i}-\mu\right|>\eta_{k}\right\}\right\}=\lim _{k \rightarrow \infty} P\left(\bigcup_{i=1}^{k}\left\{\left|X_{i}-\mu\right|>\eta_{k}\right\}\right) \text { a.s. }
\end{aligned}
$$

Proof The relations (2.11)-(2.13) follow by the same method as in the proof of Lemma 2.2, and the details are omitted here.

To prove that under the hypotheses of Theorem 1.1, the relations (2.2) and (2.8)-(2.10) hold true, we show them by using the following four lemmas.

Lemma 2.4 Assume that $f$ is a nonnegative, bounded Lipschitz function such that $f(x) \leq C$ and $|f(x)-f(y)| \leq C|x-y|$ for every $x, y \in R$. Then, under the assumptions of Theorem 1.1, there exists a positive constant $\epsilon$ such that

$$
\operatorname{Var}\left(\sum_{k=1}^{n} d_{k} f\left(\frac{\tilde{Y}_{k}}{\beta \sqrt{2 k l\left(\eta_{k}\right)}}\right)\right) \ll D_{n}^{2}\left(\ln D_{n}\right)^{-1-\epsilon}
$$


Proof Write

$$
\begin{aligned}
& \operatorname{Var}\left(\sum_{i=1}^{n} d_{i} f\left(\frac{\tilde{Y}_{i}}{\beta \sqrt{2 i l\left(\eta_{i}\right)}}\right)\right) \\
& \quad \leq 2\left(\sum_{1 \leq i \leq j \leq(2 i) \wedge n}+\sum_{1 \leq 2 i<j \leq n}\right) d_{i} d_{j}\left|\operatorname{Cov}\left(f\left(\frac{\tilde{Y}_{i}}{\beta \sqrt{2 i l\left(\eta_{i}\right)}}\right), f\left(\frac{\tilde{Y}_{j}}{\beta \sqrt{2 j l\left(\eta_{j}\right)}}\right)\right)\right| \\
& \quad=: I_{1}+I_{2} .
\end{aligned}
$$

From (2.6), we get

$$
\ln D_{n} \sim \ln ^{\alpha} n, \quad \exp \left(\ln ^{\alpha} n\right) \sim \frac{D_{n}}{\left(\ln D_{n}\right)^{(1-\alpha) / \alpha}} .
$$

Since $f$ is a nonnegative, bounded Lipschitz function, it follows from (2.16) that for any $0<\epsilon<(1-2 \alpha) / \alpha$ with $0 \leq \alpha<1 / 2$,

$$
I_{1} \leq C \sum_{1 \leq i \leq j \leq(2 i) \wedge n} d_{i} d_{j} \leq C \frac{D_{n}^{2}}{\left(\ln D_{n}\right)^{(1-\alpha) / \alpha}} \sum_{j=i}^{2 i} \frac{1}{j} \ll D_{n}^{2}\left(\ln D_{n}\right)^{-1-\epsilon} .
$$

Consider $I_{2}$ now. Let $\widetilde{Y}_{2 i, j}=\sum_{k=2 i+1}^{j} b_{k, j} \tilde{X}_{k j}^{*}=\sum_{k=2 i+1}^{j} \sum_{l=k}^{j} \frac{1}{l} \tilde{X}_{k j}^{*}$ for $1 \leq 2 i<j=3,4, \ldots$, then

$$
\begin{aligned}
& \left|\operatorname{Cov}\left(f\left(\frac{\tilde{Y}_{i}}{\beta \sqrt{2 i l\left(\eta_{i}\right)}}\right), f\left(\frac{\tilde{Y}_{j}}{\beta \sqrt{2 j l\left(\eta_{j}\right)}}\right)\right)\right| \\
& \leq\left|\operatorname{Cov}\left(f\left(\frac{\tilde{Y}_{i}}{\beta \sqrt{2 i l\left(\eta_{i}\right)}}\right), f\left(\frac{\widetilde{Y}_{2 i, j}}{\beta \sqrt{2 j l\left(\eta_{j}\right)}}\right)\right)\right| \\
& \quad+\mid E f\left(\frac{\tilde{Y}_{i}}{\beta \sqrt{2 i l\left(\eta_{i}\right)}}\right)\left(f\left(\frac{\tilde{Y}_{j}}{\beta \sqrt{2 j l\left(\eta_{j}\right)}}\right)-f\left(\frac{\tilde{Y}_{2 i, j}}{\beta \sqrt{2 j l\left(\eta_{j}\right)}}\right)\right) \\
& \quad-E f\left(\frac{\tilde{Y}_{i}}{\beta \sqrt{2 i l\left(\eta_{i}\right)}}\right) E\left(f\left(\frac{\tilde{Y}_{j}}{\beta \sqrt{2 j l\left(\eta_{j}\right)}}\right)-f\left(\frac{\tilde{Y}_{2 i, j}}{\beta \sqrt{2 j l\left(\eta_{j}\right)}}\right)\right) \mid=: I_{21}+I_{22} .
\end{aligned}
$$

The well-known property of a $\phi$-mixing sequence (see [17, Lemma 1.2.9]) and the boundedness of $f$ imply $\left|I_{21}\right| \leq C \phi(i)$. Since $\sum_{n \geq 1} \phi^{1 / 2}\left(2^{n}\right)<\infty$ implies $\phi(n) \ll(\ln n)^{-1}$, it follows that for any $0<\epsilon<(1-2 \alpha) / \alpha$ with $0 \leq \alpha<1 / 2$,

$$
\sum_{1 \leq 2 i<j \leq n} d_{i} d_{j} I_{21} \leq C \frac{D_{n}^{2}}{\left(\ln D_{n}\right)^{(1-\alpha) / \alpha}} \sum_{i=1}^{n} \frac{1}{i \ln i} \ll D_{n}^{2}\left(\ln D_{n}\right)^{-1-\epsilon}
$$

Estimate $I_{22}$. Since $\left\{X_{n}\right\}_{n \geq 1}$ is stationary and $\sum_{n=1}^{\infty} \phi^{1 / 2}\left(2^{n}\right)<\infty$, it follows from the relation (2.2) in Li and Wang [6] that

$$
\begin{aligned}
E\left(\sum_{k=1}^{2 i} b_{k, j} \tilde{X}_{k j}^{*}\right)^{2} & =\sum_{k=1}^{2 i} b_{k, j}^{2} E\left(\widetilde{X}_{k j}^{*}\right)^{2}+2 \sum_{k=1}^{2 i-1} \sum_{l=k+1}^{2 i} b_{k, j} b_{l, j} E \tilde{X}_{k j}^{*} \widetilde{X}_{l j}^{*} \\
& =\sum_{k=1}^{2 i} b_{k, j}^{2} E\left(\tilde{X}_{k j}^{*}\right)^{2}+2 \sum_{k=2}^{2 i} \sum_{l=1}^{2 i} b_{l, j}^{2} E \tilde{X}_{1 j}^{*} \tilde{X}_{k j}^{*}-2 \sum_{k=2}^{2 i} \sum_{l=2 i-k+2}^{2 i} b_{l, j}^{2} E \tilde{X}_{1 j}^{*} \tilde{X}_{k j}^{*}
\end{aligned}
$$




$$
\begin{aligned}
& -2 \sum_{k=2}^{2 i} \sum_{l=1}^{2 i+1-k} b_{l, j} b_{l, l+k-2} E \widetilde{X}_{l j}^{*} \widetilde{X}_{k j}^{*} \\
\leq & \sum_{k=1}^{2 i} b_{k, j}^{2}\left(E\left(\widetilde{X}_{k j}^{*}\right)^{2}+6 \sum_{l=2}^{2 i}\left|E \widetilde{X}_{1 j}^{*} \widetilde{X}_{l j}^{*}\right|\right) \\
\leq & \sum_{k=1}^{2 i} b_{k, j}^{2}\left(l\left(\eta_{j}\right)+C l\left(\eta_{j}\right) \sum_{l=1}^{\infty} \phi^{1 / 2}(l)\right) \\
\leq & C l\left(\eta_{j}\right) \sum_{k=1}^{2 i} b_{k, j}^{2}
\end{aligned}
$$

by using Lemma 1.2.8 in Lin and Lu [17]. Note that for $n \geq k, \sum_{i=1}^{k} \log ^{2}(n / i) \leq C k(1+$ $\left.\log ^{2}(n / k)\right)$. Using the fact that $\left\{X_{n}\right\}_{n \geq 1}$ is stationary and that $f$ is bounded and Lipschitzian, we get

$$
\begin{aligned}
I_{22} & \leq C \frac{E\left|\sum_{k=1}^{2 i} b_{k, j} \widetilde{X}_{k j}^{*}\right|}{\beta \sqrt{2 j l\left(\eta_{j}\right)}} \\
& \leq \frac{C}{\sqrt{2 j l\left(\eta_{j}\right)}}\left(E\left|\sum_{k=1}^{2 i} b_{k, j} \widetilde{X}_{k j}^{*}\right|^{2}\right)^{1 / 2} \\
& \leq C \frac{\sqrt{l\left(\eta_{j}\right)}}{\sqrt{j l\left(\eta_{j}\right)}}\left(\sum_{k=1}^{2 i}\left(\sum_{l=k}^{j} \frac{1}{l}\right)^{2}\right)^{1 / 2} \\
& \leq \frac{C}{\sqrt{j}}\left(\sum_{k=1}^{2 i} \log ^{2}\left(\frac{j}{k}\right)\right)^{1 / 2} \\
& \leq C \frac{\sqrt{2 i}}{\sqrt{j}}\left(1+\log ^{2}(j /(2 i))\right)^{1 / 2} \\
& \leq C \frac{\sqrt{2 i}}{\sqrt{j}}(1+\log (j /(2 i))) \\
& \leq C(2 i / j)^{\delta},
\end{aligned}
$$

where $\delta \in(0,1 / 2)$. It follows that

$$
\begin{aligned}
\sum_{1 \leq 2 i<j \leq n} d_{i} d_{j} I_{22} & \leq \sum_{\substack{1 \leq 2 i<j \leq n \\
j /(2 i) \geq\left(\ln D_{n}\right)^{2 / \delta}}} d_{i} d_{j}\left(\frac{2 i}{j}\right)^{\delta}+C \sum_{\substack{1 \leq 2 i<j \leq n \\
j /(2 i) \leq\left(\ln D_{n}\right)^{2 / \delta}}} d_{i} d_{j}\left(\frac{2 i}{j}\right)^{\delta} \\
& \leq\left(\ln D_{n}\right)^{-2} \sum_{i=1}^{n} d_{i} \sum_{j=1}^{n} d_{j}+C \sum_{i=1}^{n} d_{i} \sum_{j=2 i}^{\left[2 i\left(\ln D_{n}\right)^{2 / \delta}\right]} d_{j} \\
& \leq C D_{n}^{2}\left(\ln D_{n}\right)^{-2}+C \exp (\ln \alpha) \sum_{i=1}^{n} d_{i} \sum_{j=2 i}^{\left[2 i\left(\ln D_{n}\right)^{2 / \delta}\right]} \frac{1}{j} \\
& \leq C D_{n}^{2}\left(\ln D_{n}\right)^{-2}+C D_{n}^{2} \frac{\ln \ln D_{n}}{\left(\ln D_{n}\right)^{(1-\alpha) / \alpha}} \\
& \leq C D_{n}^{2}\left(\ln D_{n}\right)^{-1-\epsilon}
\end{aligned}
$$


for any $0<\epsilon<(1-2 \alpha) / \alpha$ with $0 \leq \alpha<1 / 2$. From (2.18) and (2.19), we get

$$
J_{2} \leq C D_{n}^{2}\left(\ln D_{n}\right)^{-1-\epsilon} .
$$

Hence, combining (2.15) with (2.17) and (2.20) yields (2.14).

Lemma 2.5 Under the hypotheses of Lemma 2.4, there exists a positive constant $\epsilon$ such that

$$
\operatorname{Var}\left(\sum_{k=1}^{n} d_{k} f\left(\frac{\widehat{Y}_{k}}{\beta \sqrt{2 k l\left(\eta_{k}\right)}}\right)\right) \ll D_{n}^{2}\left(\ln D_{n}\right)^{-1-\epsilon}
$$

Proof By the same method as in the proof of Lemma 2.4, we show (2.21). We have

$$
\begin{aligned}
& \operatorname{Var}\left(\sum_{i=1}^{n} d_{i} f\left(\frac{\widehat{Y}_{i}}{\beta \sqrt{2 i l\left(\eta_{i}\right)}}\right)\right) \\
& \quad \leq 2\left(\sum_{1 \leq i \leq j \leq(2 i) \wedge n}+\sum_{1 \leq 2 i<j \leq n}\right) d_{i} d_{j}\left|\operatorname{Cov}\left(f\left(\frac{\widehat{Y}_{i}}{\beta \sqrt{2 i l\left(\eta_{i}\right)}}\right), f\left(\frac{\widehat{Y}_{j}}{\beta \sqrt{2 j l\left(\eta_{j}\right)}}\right)\right)\right|=: J_{1}+J_{2} .
\end{aligned}
$$

In the same manner as in (2.17), we can see that $J_{1} \leq C D_{n}^{2}\left(\ln D_{n}\right)^{-1-\epsilon}$. Consider $J_{2}$ now. Let $\widehat{Y}_{2 i, j}=\sum_{k=2 i+1}^{j} b_{k, j} \widehat{X}_{k j}^{*}=\sum_{k=2 i+1}^{j} \sum_{l=k}^{j} \frac{1}{l} \widehat{X}_{k j}^{*}$ for $1 \leq 2 i<j=3,4, \ldots$, then

$$
\begin{aligned}
\left|\operatorname{Cov}\left(f\left(\frac{\widehat{Y}_{i}}{\beta \sqrt{2 i l\left(\eta_{i}\right)}}\right), f\left(\frac{\widehat{Y}_{j}}{\beta \sqrt{2 j l\left(\eta_{j}\right)}}\right)\right)\right| \\
\leq\left|\operatorname{Cov}\left(f\left(\frac{\widehat{Y}_{i}}{\beta \sqrt{2 i l\left(\eta_{i}\right)}}\right), f\left(\frac{\widehat{Y}_{2 i, j}}{\beta \sqrt{2 j l\left(\eta_{j}\right)}}\right)\right)\right| \\
\quad+\mid E f\left(\frac{\widehat{Y}_{i}}{\beta \sqrt{i l\left(\eta_{i}\right)}}\right)\left(f\left(\frac{\widehat{Y}_{j}}{\beta \sqrt{2 j l\left(\eta_{j}\right)}}\right)-f\left(\frac{\widehat{Y}_{2 i, j}}{\beta \sqrt{2 j l\left(\eta_{j}\right)}}\right)\right) \\
\quad-E f\left(\frac{\widehat{Y}_{i}}{\beta \sqrt{2 i l\left(\eta_{i}\right)}}\right) E\left(f\left(\frac{\widehat{Y}_{j}}{\beta \sqrt{2 j l\left(\eta_{j}\right)}}\right)-f\left(\frac{\widehat{Y}_{2 i, j}}{\beta \sqrt{2 j l\left(\eta_{j}\right)}}\right)\right) \mid=: J_{21}+J_{22} .
\end{aligned}
$$

As in (2.18), we can see that $\sum_{1 \leq 2 i<j \leq n} d_{i} d_{j} J_{21} \ll D_{n}^{2}\left(\ln D_{n}\right)^{-1-\epsilon}$. Estimate $J_{22}$. By Lemma 2.1 and $\eta_{j}^{2} \sim j l\left(\eta_{j}\right)$, there exists $j_{0}$ such that $E\left|X_{1}-\mu\right| I\left\{\left|X_{1}-\mu\right|>\eta_{j}\right\} \leq l\left(\eta_{j}\right) / \eta_{j}$ for every $j>j_{0}$. Using the fact that $\left\{X_{n}\right\}_{n \geq 1}$ is stationary and that $f$ is bounded and Lipschitzian, we get

$$
\begin{aligned}
J_{22} & \leq C \frac{E\left|\sum_{k=1}^{2 i} b_{k, j} \widehat{X}_{k j}^{*}\right|}{\beta \sqrt{2 j l\left(\eta_{j}\right)}} \leq C \frac{E\left|\widehat{X}_{1 j}^{*}\right|}{\sqrt{2 j l\left(\eta_{j}\right)}} \sum_{k=1}^{2 i} b_{k, j} \\
& \leq C \frac{E\left|X_{1}-\mu\right| I\left\{\left|X_{1}-\mu\right|>\eta_{j}\right\}}{\sqrt{2 j l\left(\eta_{j}\right)}}\left(\sum_{k=1}^{2 i} \sum_{l=k}^{2 i} \frac{1}{l}+2 i b_{2 i+1, j}\right) \\
& \leq \frac{C}{\sqrt{2 j l\left(\eta_{j}\right)}} \frac{l\left(\eta_{j}\right)}{\eta_{j}}(2 i+2 i \log (j /(2 i))) \\
& \leq C\left(\frac{2 i}{j}\right)^{\delta}
\end{aligned}
$$


for large enough $i$ with $2 i<j$, where $\delta \in(0,1)$, since for any $\gamma>0, \log n \leq n^{\gamma}$ for large $n$. Similarly, we get by $(2.19)$

$$
\sum_{1 \leq 2 i<j \leq n} d_{i} d_{j} J_{22} \ll D_{n}^{2}\left(\ln D_{n}\right)^{-1-\epsilon}
$$

which means $J_{2} \leq C D_{n}^{2}\left(\ln D_{n}\right)^{-1-\epsilon}$, and hence (2.21) is proved.

Lemma 2.6 Under the hypotheses of Lemma 2.4, there exists a positive constant $\epsilon$ such that

$$
\operatorname{Var}\left(\sum_{k=1}^{n} d_{k} f\left(\frac{\widetilde{V}_{k}^{2}}{k l\left(\eta_{k}\right)}\right)\right) \ll D_{n}^{2}\left(\ln D_{n}\right)^{-1-\epsilon}
$$

Proof This follows by the same method as the proof of Lemma 2.4, and the details are omitted.

Lemma 2.7 Under the hypotheses of Theorem 2.4, there exists a positive constant $\epsilon$ such that

$$
\operatorname{Var}\left(\sum_{i=1}^{n} d_{i} I\left\{\bigcup_{k=1}^{i}\left\{\left|X_{k}-\mu\right|>\eta_{i}\right\}\right\}\right) \ll D_{n}^{2}\left(\ln D_{n}\right)^{1-\epsilon} .
$$

Proof We have divided the proof into three parts:

$$
\begin{aligned}
& \operatorname{Var}\left(\sum_{i=1}^{n} d_{i} I\left\{\bigcup_{k=1}^{i}\left\{\left|X_{k}-\mu\right|>\eta_{k}\right\}\right\}\right) \\
& \leq \sum_{i=1}^{n} d_{i}^{2} \operatorname{Var}\left(I\left\{\bigcup_{k=1}^{i}\left\{\left|X_{k}-\mu\right| \geq \eta_{k}\right\}\right\}\right)+2\left(\sum_{1 \leq i<j \leq(2 i) \wedge n}+\sum_{1 \leq 2 i<j \leq n}\right) d_{i} d_{j} \\
& \quad \times\left|\operatorname{Cov}\left(I\left\{\bigcup_{k=1}^{i}\left\{\left|X_{k}-\mu\right|>\eta_{k}\right\}\right\}, I\left\{\bigcup_{k=1}^{j}\left\{\left|X_{k}-\mu\right|>\eta_{k}\right\}\right\}\right)\right| \\
& =: L_{1}+L_{2}+L_{3} .
\end{aligned}
$$

It is clear from (2.7) and (2.17) that

$$
L_{1} \leq \sum_{i=1}^{n} \frac{\exp \left(2 \ln ^{\alpha} i\right)}{i^{2}} \leq C, \quad L_{2} \ll D_{n}^{2}\left(\ln D_{n}\right)^{-1-\epsilon} .
$$

Consider $L_{3}$ now. It is clear that $I(E \cup F)-I(F) \leq I(E)$ for any sets $E$ and $F$, then we note that for $1 \leq 2 i<j \leq n$,

$$
\begin{aligned}
& \left|\operatorname{Cov}\left(I\left\{\bigcup_{k=1}^{i}\left\{\left|X_{k}-\mu\right| \geq \eta_{i}\right\}\right\}, I\left\{\bigcup_{k=1}^{j}\left\{\left|X_{k}-\mu\right| \geq \eta_{j}\right\}\right\}\right)\right| \\
& \leq\left|\operatorname{Cov}\left(I\left\{\bigcup_{k=1}^{i}\left\{\left|X_{k}-\mu\right| \geq \eta_{i}\right\}\right\}, I\left\{\bigcup_{k=2 i+1}^{j}\left\{\left|X_{k}-\mu\right| \geq \eta_{j}\right\}\right\}\right)\right|
\end{aligned}
$$




$$
\begin{aligned}
& +\mid E I\left\{\bigcup_{k=1}^{i}\left\{\left|X_{k}-\mu\right| \geq \eta_{i}\right\}\right\}\left(I\left\{\bigcup_{k=1}^{j}\left\{\left|X_{k}-\mu\right| \geq \eta_{j}\right\}\right\}-I\left\{\bigcup_{k=2 i+1}^{j}\left\{\left|X_{k}-\mu\right| \geq \eta_{j}\right\}\right\}\right) \\
& -E I\left\{\bigcup_{k=1}^{i}\left\{\left|X_{k}-\mu\right| \geq \eta_{i}\right\}\right\} \\
& \times E\left(I\left\{\bigcup_{k=1}^{j}\left\{\left|X_{k}-\mu\right| \geq \eta_{j}\right\}\right\}-I\left\{\bigcup_{k=2 i+1}^{j}\left\{\left|X_{k}-\mu\right| \geq \eta_{j}\right\}\right\}\right) \mid \\
& \leq\left|\operatorname{Cov}\left(I\left\{\bigcup_{k=1}^{i}\left\{\left|X_{k}-\mu\right| \geq \eta_{i}\right\}\right\}, I\left\{\bigcup_{k=2 i+1}^{j}\left\{\left|X_{k}-\mu\right| \geq \eta_{j}\right\}\right\}\right)\right| \\
& +2 C\left|E I\left\{\bigcup_{k=1}^{2 i}\left\{\left|X_{k}-\mu\right| \geq \eta_{j}\right\}\right\}\right| .
\end{aligned}
$$

From the property of a $\phi$-mixing sequence and $\phi(i) \ll(\log i)^{-1}$, we have

$$
\left|\operatorname{Cov}\left(I\left\{\bigcup_{k=1}^{i}\left\{\left|X_{k}-\mu\right| \geq \eta_{i}\right\}\right\}, I\left\{\bigcup_{k=2 i+1}^{j}\left\{\left|X_{k}-\mu\right| \geq \eta_{j}\right\}\right\}\right)\right| \leq C \phi(i)
$$

and hence

$$
\begin{aligned}
\sum_{1 \leq 2 i<j \leq n} d_{i} d_{j} \phi(i) & \leq C \frac{D_{n}^{2}}{\left(\ln D_{n}\right)^{(1-\alpha) / \alpha}} \sum_{i=1}^{n} \frac{1}{i \ln i} \\
& \leq C \frac{D_{n}^{2} \ln \ln n}{\left(\ln D_{n}\right)^{(1-\alpha) / \alpha}} \ll D_{n}^{2}\left(\ln D_{n}\right)^{-1-\epsilon}
\end{aligned}
$$

for any $0<\epsilon<(1-2 \alpha) / \alpha$. By the stationarity of $\left\{X_{n}\right\}_{n \geq 1}$ and Lemma 2.2(b), we get $\sum_{k=1}^{n} P\left\{\left|X_{k}-\mu\right| \geq \eta_{n}\right\}=n P\left\{\left|X_{1}-\mu\right| \geq \eta_{n}\right\}=o(1)$, which yields $E I\left\{\bigcup_{k=1}^{2 i}\left\{\left|X_{k}-\mu\right| \geq \eta_{j}\right\}\right\} \leq$ $\sum_{k=1}^{2 i} P\left\{\left|X_{k}-\mu\right| \geq \eta_{j}\right\}=2 i P\left\{\left|X_{1}-\mu\right| \leq \eta_{j}\right\} \ll 2 i / j$, and hence, in the same way as in (2.19),

$$
\sum_{1 \leq 2 i<j \leq n} d_{i} d_{j} \sum_{k=1}^{2 i} P\left\{\left|X_{k}-\mu\right| \geq \eta_{j}\right\} \leq C D_{n}^{2}\left(\ln D_{n}\right)^{-1-\epsilon}
$$

From (2.25) and (2.26), it follows that

$$
L_{3} \ll D_{n}^{2}\left(\ln D_{n}\right)^{-1-\epsilon}
$$

Therefore, combining (2.23) with (2.24) and (2.27), we obtain (2.22), which is our claim.

\section{Proof of Theorem 1.1}

Let $C_{i}=S_{i} /(i \mu)$. To prove Theorem 1.1, it suffices to show that

$$
\lim _{n \rightarrow \infty} \frac{1}{D_{n}} \sum_{k=1}^{n} d_{k} I\left\{\frac{\mu}{\beta \sqrt{2} V_{k}} \sum_{i=1}^{k} \log C_{i} \leq x\right\}=\Phi(x) \quad \text { a.s. }
$$


for any $x \in R$. For any given $0<\epsilon<1$, it is clear that

$$
\begin{aligned}
& I\left\{\frac{\mu}{\beta \sqrt{2} V_{k}} \sum_{i=1}^{k} \log C_{i} \leq x\right\} \\
& \quad \leq \max \left\{I\left\{\frac{\mu \sum_{i=1}^{k} \log C_{i}}{\beta \sqrt{2(1+\epsilon) k l\left(\eta_{k}\right)}} \leq x\right\}+I\left\{\tilde{V}_{k}^{2}>(1+\epsilon) k l\left(\eta_{k}\right)\right\},\right. \\
& \left.\quad I\left\{\frac{\mu \sum_{i=1}^{k} \log C_{i}}{\beta \sqrt{2(1-\epsilon) k l\left(\eta_{k}\right)}} \leq x\right\}+I\left\{\tilde{V}_{k}^{2}<(1-\epsilon) k l\left(\eta_{k}\right)\right\}\right\}+I\left\{\bigcup_{i=1}^{k}\left\{\left|X_{i}-\mu\right|>\eta_{k}\right\}\right\}
\end{aligned}
$$

and

$$
\begin{aligned}
& I\left\{\frac{\mu}{\beta \sqrt{2} V_{k}} \sum_{i=1}^{k} \log C_{i} \leq x\right\} \\
& \quad \geq \min \left\{I\left\{\frac{\mu \sum_{i=1}^{k} \log C_{i}}{\beta \sqrt{2(1-\epsilon) k l\left(\eta_{k}\right)}} \leq x\right\}-I\left\{\widetilde{V}_{k}^{2}<(1-\epsilon) k l\left(\eta_{k}\right)\right\},\right. \\
& \left.\quad I\left\{\frac{\mu \sum_{i=1}^{k} \log C_{i}}{\beta \sqrt{2(1+\epsilon) k l\left(\eta_{k}\right)}} \leq x\right\}-I\left\{\widetilde{V}_{k}^{2}>(1+\epsilon) k l\left(\eta_{k}\right)\right\}\right\}-I\left\{\bigcup_{i=1}^{k}\left\{\left|X_{i}-\mu\right|>\eta_{k}\right\}\right\} .
\end{aligned}
$$

Hence it suffices to show

$$
\begin{aligned}
& \frac{1}{D_{n}} \sum_{k=1}^{n} d_{k} I\left\{\frac{\mu \sum_{i=1}^{k} \log C_{i}}{\beta \sqrt{2 k l\left(\eta_{k}\right)}} \leq x\right\} \rightarrow \Phi(\sqrt{1 \pm \epsilon} \cdot x) \quad \text { a.s., } \\
& \frac{1}{D_{n}} \sum_{k=1}^{n} d_{k} I\left\{\bigcup_{i=1}^{k}\left\{\left|X_{i}-\mu\right|>\eta_{k}\right\}\right\} \rightarrow 0 \quad \text { a.s., } \\
& \frac{1}{D_{n}} \sum_{k=1}^{n} d_{k} I\left\{\tilde{V}_{k}^{2}>(1+\epsilon) k l\left(\eta_{k}\right)\right\} \rightarrow 0 \quad \text { a.s., } \\
& \frac{1}{D_{n}} \sum_{k=1}^{n} d_{k} I\left\{\tilde{V}_{k}^{2}<(1-\epsilon) k l\left(\eta_{k}\right)\right\} \rightarrow 0 \text { a.s. }
\end{aligned}
$$

Let $0<\delta<1 / 2$ and $f$ be a real function such that for any given $x \in R$,

$$
I\{y \leq \sqrt{1 \pm \epsilon} \cdot x-\delta\} \leq f_{x}(y)=f(y) \leq I\{\sqrt{1 \pm \epsilon} \cdot x+\delta\}
$$

We first prove that (3.1) holds under condition (2.2). Note that $E|X|^{p}<\infty$ for all $1<p<2$ since $X$ belongs to the domain of attraction of the normal law. For our purpose, we fix $4 / 3<p<2$. By the Marcinkiewicz-Zygmund strong law of a large number for $\phi$-mixing sequences (see [17, Remark 8.2.1], [18]), for $i$ large enough, we have

$$
\left|C_{i}-1\right| \leq i^{1 / p}-1 \quad \text { a.s. }
$$

It is easy to see that $\log (1+x)-x=O\left(x^{2}\right)$ as $x \rightarrow 0$. Thus

$$
\left|\sum_{i=1}^{k} \log C_{i}-\sum_{i 1}^{k}\left(C_{i}-1\right)\right| \ll \sum_{i=1}^{k}\left(C_{i}-1\right)^{2} \ll k^{2 / p-1} \quad \text { a.s. }
$$


Hence for almost every event $\omega$ and any $0<\delta_{1}<1 / 4$, there exists $k_{0}=k_{0}\left(\omega, \delta_{1}, x\right)$ such that for $k>k_{0}$,

$$
\begin{aligned}
I\left\{\frac{\mu \sum_{i=1}^{k}\left(C_{i}-1\right)}{\beta \sqrt{2 k l\left(\eta_{k}\right)}} \leq \sqrt{1 \pm \epsilon} \cdot x-\delta_{1}\right\} & \leq I\left\{\frac{\mu \sum_{i=1}^{k} \log C_{i}}{\beta \sqrt{2 k l\left(\eta_{k}\right)}} \leq \sqrt{1 \pm \epsilon} \cdot x\right\} \\
& \leq I\left\{\frac{\mu \sum_{i=1}^{k}\left(C_{i}-1\right)}{\beta \sqrt{2 k l\left(\eta_{k}\right)}} \leq \sqrt{1 \pm \epsilon} \cdot x+\delta_{1}\right\} .
\end{aligned}
$$

We note that

$$
\mu \sum_{i=1}^{k}\left(C_{i}-1\right)=\sum_{j=1}^{k} \sum_{l=j}^{k} \frac{1}{l} \widetilde{X}_{j k}^{*}+\sum_{j=1}^{k} \sum_{l=j}^{k} \frac{1}{l} \widehat{X}_{j k}^{*}=\widetilde{Y}_{k}+\widehat{Y}_{k} .
$$

So, for any $0<\delta_{2}<1 / 4$, we have

$$
\begin{aligned}
I\left\{\frac{\mu \sum_{i=1}^{k}\left(C_{i}-1\right)}{\left.\beta \sqrt{2 k l\left(\eta_{k}\right)} \leq \sqrt{1 \pm \epsilon} \cdot x+\delta_{1}\right\} \leq}\right. & I\left\{\frac{\widetilde{Y}_{k}}{\beta \sqrt{2 k l\left(\eta_{k}\right)}} \leq \sqrt{1 \pm \epsilon} \cdot x+\delta_{1}+\delta_{2}\right\} \\
& +I\left\{\frac{\left|\widehat{Y}_{k}\right|}{\beta \sqrt{2 k l\left(\eta_{k}\right)}}>\delta_{2}\right\}
\end{aligned}
$$

and

$$
\begin{aligned}
I\left\{\frac{\mu \sum_{i=1}^{k}\left(C_{i}-1\right)}{\beta \sqrt{2 k l\left(\eta_{k}\right)}} \leq \sqrt{1 \pm \epsilon} \cdot x-\delta_{1}\right\} \geq & I\left\{\frac{\widetilde{Y}_{k}}{\beta \sqrt{2 k l\left(\eta_{k}\right)}} \leq \sqrt{1 \pm \epsilon} \cdot x-\delta_{1}-\delta_{2}\right\} \\
& -I\left\{\frac{\left|\widehat{Y}_{k}\right|}{\beta \sqrt{2 k l\left(\eta_{k}\right)}}>\delta_{2}\right\} .
\end{aligned}
$$

Let $\lambda=\delta_{2} \beta \sqrt{2}$ with $0<\delta_{2}<1 / 4$. By using the fact that $\left\{X_{k}\right\}_{k \geq 1}$ is stationary and Lemma 2.1(c), we have

$$
\begin{aligned}
P\left\{\left|\widehat{Y}_{k}\right| \geq \lambda \sqrt{k l\left(\eta_{k}\right)}\right\} & \leq P\left\{\sum_{i=1}^{k} b_{i, k}\left|\widehat{X}_{1 k}^{*}\right| \geq \lambda \sqrt{k l\left(\eta_{k}\right)}\right\} \leq \frac{\left(\sum_{i=1}^{k} b_{i, k}\right) E\left|\widehat{X}_{1 k}^{*}\right|}{\lambda \sqrt{k l\left(\eta_{k}\right)}} \\
& \leq \frac{2 k E\left|X_{1}-\mu\right| I\left\{\left|X_{1}-\mu\right| \geq \eta_{k}\right\}}{\lambda \sqrt{k l\left(\eta_{k}\right)}}=o(1),
\end{aligned}
$$

and by (2.3) in Lemma 2.2, we get

$$
\frac{1}{D_{n}} \sum_{k=1}^{n} d_{k} I\left\{\frac{\widetilde{Y}_{k}}{\beta \sqrt{2 k l\left(\eta_{k}\right)}} \leq x\right\} \rightarrow \Phi\left(\sqrt{1 \pm \epsilon} \cdot x \pm \delta_{1} \pm \delta_{2}\right) \quad \text { a.s. }
$$

for any $x \in R$. Hence, combining (3.5)-(3.9) yields (3.1) by the arbitrariness of $\delta_{1}, \delta_{2}$. For (3.2), it is clear from (2.13) in Lemma 2.3 that (3.2) holds true since $P\left(\bigcup_{i=1}^{k}\left\{\left|X_{i}-\mu\right| \geq\right.\right.$ $\left.\left.\eta_{k}\right\}\right) \leq k P\left\{\left|X_{1}-\mu\right| \geq \eta_{k}\right\}=o(1)$. Consider (3.3). By (2.12) in Lemma 2.3, it suffices to show that

$$
P\left\{\widetilde{V}_{k}^{2}>(1+\epsilon) k l\left(\eta_{k}\right)\right\} \rightarrow 0 \quad \text { as } k \rightarrow \infty
$$


We note that $\left\{\widetilde{X}_{j k}^{2}-E \widetilde{X}_{j k}^{2}\right\}_{j=1}^{k}$ is a $\phi$-mixing sequence with the same mixing coefficient $\phi(k)$. Using again Lemma 2.3 in Shao [19] and Lemma 1(d), we obtain

$$
\eta_{k}^{-4} E\left(\sum_{j=1}^{k}\left(\tilde{X}_{j k}^{2}-E \tilde{X}_{j k}^{2}\right)\right)^{2} \leq C k \eta_{k}^{-4} \max _{1 \leq j \leq k} E\left(\tilde{X}_{j k}^{2}-E \tilde{X}_{j k}^{2}\right)^{2} \leq C k \eta_{k}^{-4} E \tilde{X}_{1 k}^{4}=o(1) .
$$

Hence, by Chebyshev's inequality and again recalling $\eta_{k}^{2} \sim k l\left(\eta_{k}\right)$, we have

$$
P\left\{\left|\tilde{V}_{k}^{2}-E \tilde{V}_{k}^{2}\right|>\epsilon k l\left(\eta_{k}\right)\right\} \leq \frac{E\left|\tilde{V}_{k}^{2}-E \tilde{V}_{k}^{2}\right|^{2}}{\epsilon^{2}\left(k l\left(\eta_{k}\right)\right)^{2}} \leq C \epsilon^{-2} \eta_{k}^{-4} E\left(\sum_{j=1}^{k}\left(\tilde{X}_{j k}^{2}-E \tilde{X}_{j k}^{2}\right)\right)^{2}=o(1)
$$

and $E \widetilde{V}_{k}^{2}=\sum_{i=1}^{k} l\left(\eta_{i}\right) \sim k l\left(\eta_{k}\right)$, which implies that

$$
P\left\{\widetilde{V}_{k}^{2}>(1+\epsilon) k l\left(\eta_{k}\right)\right\} \leq P\left\{\widetilde{V}_{k}^{2}-E \widetilde{V}_{k}^{2}>\frac{\epsilon}{2} k l\left(\eta_{k}\right)\right\}=o(1)
$$

and hence (3.3) holds true. Similarly,

$$
P\left\{\tilde{V}_{k}^{2}<(1-\epsilon) k l\left(\eta_{k}\right)\right\}=o(1),
$$

which implies that (3.4). The proof is completed.

\section{Competing interests}

The author did not provide this information.

Received: 21 December 2012 Accepted: 12 March 2013 Published: 4 April 2013

\section{References}

1. Brosamler, GA: An almost everywhere central limit theorem. Math. Proc. Camb. Philos. Soc. 104, 561-574 (1988)

2. Schatte, P: On strong versions of the central limit theorem. Math. Nachr. 137, 249-256 (1988)

3. Berkes, I: Results and problems related to the pointwise central limit theorem. In: Szyszkowicz, B (ed.) Asymptotic Results in Probability and Statistics (A Volume in Honor of Miklós Csörgő), pp. 59-60. Elsevier, Amsterdam (1998)

4. Gonchigdanzan, K, Rempala, GA: A note on the almost sure limit theorem for the product of partial sums. Appl. Math. Lett. 19, 191-196 (2006)

5. Gonchigdanzan, K: An almost sure limit theorem for the product of partial sums with stable distribution. Stat. Probab. Lett. 78, 3170-3175 (2008)

6. Li, YX, Wang, JF: An almost sure central limit theorem for products of sums under association. Stat. Probab. Lett. 78(4), 367-375 (2008)

7. Zhang, Y, Yang, XY, Dong, ZS: An almost sure central limit theorem for products of sums of partial sums under association. J. Math. Anal. Appl. 355(2), $708-716$ (2009)

8. Huang, SH, Pang, TX: An almost sure central limit theorem for self-normalized partial sums. Comput. Math. Appl. 60 2639-2644 (2010)

9. Zhang, Y, Yang, $X Y$ : An almost sure central limit theorem for self-normalized products of sums of i.i.d. random variables. J. Math. Anal. Appl. 376, 29-41 (2011)

10. Balan, RM, Kulik, R: Self-normalized weak invariance principle for mixing sequences. Tech. Rep. Ser. 417, Lab. Reas. Probab. Stat., Univ. Ottawa-Carleton Univ. (2005)

11. Balan, RM, Kulik, R: Weak invariance principle for mixing sequences in the domain of attraction of normal law. Studia Sci. Math. Hung. 46(3), 329-343 (2009)

12. de la Pena, H, Lai, TL, Shao, QM: Self-Normalized Processes. Springer, Berlin (2010)

13. Chandrasekharan, K, Minakshisundaram, S: Typical Means. Oxford University Press, Oxford (1952)

14. Csörgő, M, Szyszkowicz, B, Wang, Q: Donsker's theorem for self-normalized partial sums processes. Ann. Probab. 31(3), 1228-1240 (2003)

15. Liu, W, Lin, ZY: Asymptotics for self-normalized random products of sums for mixing sequences. Stoch. Anal. Appl. 25, 739-762 (2007)

16. Seneta, E: Regularly Varying Functions. Springer, Berlin (1976)

17. Lin, Z, Lu, C: Limit Theory for Mixing Dependent Random Variables. Kluwer Academic, Boston (1996)

18. Xue, LG: Convergence rates of the strong law of large numbers for a mixing sequence. J. Syst. Sci. Math. Sci. 14 213-221 (1994) (in Chinese)

19. Shao, QM: Almost sure invariance principle for mixing sequences of random variables. Stoch. Process. Appl. 48 319-334 (1993) 
doi:10.1186/1029-242X-2013-155

Cite this article as: Hwang: On the almost sure central limit theorem for self-normalized products of partial sums of $\phi$-mixing random variables. Journal of Inequalities and Applications 2013 2013:155.

Submit your manuscript to a SpringerOpen ${ }^{\circ}$ journal and benefit from:

- Convenient online submission

- Rigorous peer review

- Immediate publication on acceptance

- Open access: articles freely available online

- High visibility within the field

- Retaining the copyright to your article

Submit your next manuscript at $\gg$ springeropen.com 